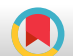

\title{
Noninvasive Models to Predict Liver Fibrosis in Patients with Chronic Hepatitis B: A Study from \\ Turkey
}

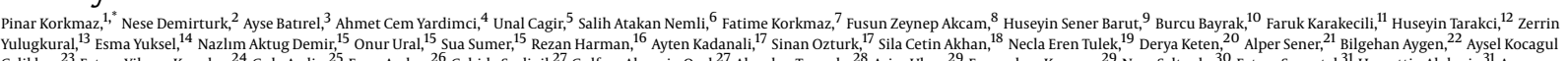

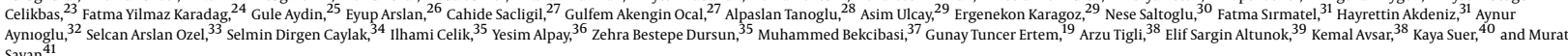
Sayan

${ }^{1}$ Dumlupinar University Faculty of Medicine, Department of Infectious Diseases and Clinical Microbiology, Kutahya, Turkey ${ }^{2}$ Afyon Kocatepe University Faculty of Medicine, Department of Infectious Diseases and Clinical Microbiology, Afyon, Turkey

${ }^{2}$ Afyon Kocatepe University Faculty of Medicine, Department of Infectious Diseases and Clinical Microbiology, Aryon, Iurkey
${ }_{\text {Kartal Dr. Lutfi Krrdar Training and Research Hospital, Department of Infectious Diseases and Clinical Microbiology, Istanbul, Turkey }}$

${ }^{4}$ Sanliurfa Training and Research Hospital, Department of Infectious Diseases and Clinical Microbiology, Sanluurfa, Turkey

${ }^{5}$ Nevsehir State Hospital, Department of Infectious Diseases and Clinical Microbiology Nevsehir Turkey

${ }^{6}$ Izmir Katip Celebi University Faculty of Medicine, Department of Infectious Diseases and Clinical Microbiology Izmir Turkey

7 Konya Training and Research Hospital, Department of Infectious Diseases and Clinical Microbiology Konya Turkey

${ }^{8}$ Suleyman Demirel University Faculty of Medicine, Department of Infectious Diseases and Clinical Microbiology Isparta Turkey

${ }^{9}$ Gaziosmanpasa University Faculty of Medicine, Department of Infectious Diseases and Clinical Microbiology, Tokat, Turkey

${ }_{10}^{9}$ Istanbul Training and Research Hospital, Department of Infectious Diseases and Clinical Microbiology, Istanbul, Turkey

${ }^{11}$ Erzincan University Faculty of Medicine, Department of Infectious Diseases and Clinical Microbiology, Erzincan, Turkey

${ }_{12}$ Izmir Metropolitan Municipality Esrefpasa State Hospital, Department of Infectious Diseases and Clinical Microbiology, Izmir, Turkey

${ }^{13}$ Trakya University Faculty of Medicine, Department of Infectious Diseases and Clinical Microbiology, Edirne, Turkey

${ }^{14}$ Karsikaya State Hospital, Department of Infectious Diseases and Clinical Microbiology, Izmir, Turkey

${ }^{15}$ Selcuk University Faculty of Medicine, Department of Infectious Diseases and Clinical Microbiology, Konya, Turkey

${ }_{16}$ Private Sanko Hospital, Department of Infectious Diseases and Clinical Microbiology, Gaziantep, Turkey

${ }^{17}$ Health Sciences University Umraniye Training and Research Hospital, Department of Infectious Diseases and Clinical Microbiology, Istanbul, Turkey

${ }^{18}$ Kocaeli University Faculty of Medicine, Department of Infectious Diseases and Clinical Microbiology

${ }^{19}$ Ankara Training and Research Hospital, Department of Infectious Diseases and Clinical Microbiology, Ankara, Turkey

${ }^{20}$ Kahramanmaras Necip Fazıl City Hospital, Department of Infectious Diseases and Clinical Microbiology

${ }^{21}$ Canakkale Onsekiz Mart University Faculty of Medicine, Department of Infectious Diseases and Clinical Microbiology, Canakkale, Turkey

${ }^{22}$ Erciyes University Faculty of Medicine, Department of Infectious Diseases and Clinical Microbiology, Kayseri, Turkey

${ }^{23}$ Ankara NumuneTraining and Research Hospital, Department of Infectious Diseases and Clinical Microbiology,Ankara, Turkey

24 Istanbul Medeniyet University Goztepe Training and Research Hospital, Department of Infectious Diseases and Clinical Microbiology, Istanbul, Turkey

${ }^{25}$ Afyon State Hospital, Department of Infectious Diseases and Clinical Microbiology, Afyon, Turkey

26 Diyarbakir Selahaddin Eyyubi State Hospital, Department of Infectious Diseases and Clinical Microbiology, Diyarbakrr, Turke

${ }_{28}^{27}$ Kartal Yavuz Selim State Hospital, Department of Infectious Diseases and Clinical Microbiology, Istanbul, Turkey

${ }^{28}$ Istanbul Sultan Abdulhamid HanTraining Hospital, Department of Gastroenterology, Istanbul, Turkey

Istanbul Sultan Abdulhamid Han Training Hospital, Department of Infectious Diseases and Clinical Microbiology, Istanbul, Turkey

31 Cerrahpasa University Faculty of Mediche, Department of Infectious Diseases and Clinical Microbiology, Istanbul, Turkey.

32 Abant Izzet Baysal University Faculty of Medicine, Department of Infectious Diseases and Clinical Microbiology, Bolu, Turk

33 Zonguldak Ataturk State Hospital, Department of Infectious Diseases and Clinical Microbiology, Zonguldak, Turkey

${ }^{34}$ Mugla Sitkı Koçman University Faculty of Medicine, Department of Infectious Diseases and Clinical Microbiology, Mugla, Turkey

35 Mugla Sith Koçman Univesity Faculy or Medicine, Department of Hifectious Diseases and Clinical Microbiology, Mug a,

${ }^{36}$ Balikesir University Faculty of Medicine Department of fectious Diseases and Clinical Microbiology Balikesir, Turkey

37 BismilstateHosptel Deper

88 Ispate

39 Bitlis State Hospital, Department of Infectious Diseases and Clinical Microbiology, Bitlis, Turkey

${ }^{40}$ Yakın Dogu University Faculty of Medicine, Department of Infectious Diseases and Clinical Microbiology, Nicosia, TRNC

${ }^{41}$ Kocaeli University Faculty of Medicine, Department of Microbiology, Kocaeli, Turkey, Near East University, Research Center of Experimental Health Sciences, Nicosia, Northern Cypru

"Corresponding author: Pinar Korkmaz, Zigana Evleri, Yunus Emre Street, 43020, GSM: 05055502260, Kutahya, Turkey. Tel/Fax: +90-2742316660;+90-2742316673, E-mail: drpinarkor@gmail.com

Received 2016 October 30; Accepted 2017 July 01.

\begin{abstract}
Background: Many noninvasive methods, including aspartate amino transaminase(AST)/alanine amino transaminase(ALT) ratio (AAR), AST-to-platelet ratio index (APRI), Bonacini cirrhosis discriminant score (CDS), fibrosis-4 (FIB4) index, and age-platelet index (API), have been described to determine the stage of hepatic fibrosis. However, these methods are developed for patients with chronic hepatitis $\mathrm{C}(\mathrm{CHC})$ and produce conflicting results in the prediction of liver fibrosis in patients with chronic hepatitis $\mathrm{B}(\mathrm{CHB})$.

Objectives: The aim of this study was to evaluate the relationship between 7 noninvasive models, including AAR, APRI, CDS, API, FIB-4, neutrophil-to-lymphocyte ratio (NLR), and red cell distribution width (RDW)-to-platelet ratio (RPR) in patients with CHB.

Methods: The study population included all patients with CHB, undergoing liver biopsy to determine HBsAg and HBV DNA positivity in more than 6 months.

Results: A total of 2520 treatment-naive CHB patients from 40 different centers were included in the study. In total, $62.6 \%$ of the patients were male, and the mean age was $40.60 \pm 12.34$ years (minimum, 18 years; maximum, 77 years). The Ishak fibrosis score was $\geq 3$ in $29.8 \%$ of the patients, indicating significant fibrosis. The mean API, APRI, CDS, NLR, FIB4, and RPR scores in the noninvasive models were significantly different between the groups with significant and low fibrosis (P < 0.05$)$. All the noninvasive models (API, APRI, AAR, CDS, NLR, RPR, and FIB4) were found to be significant in the discrimination of cirrhosis (P< 0.05$)$. In the multiple logistic regression analysis, CDS, albumin, alkaline phosphatase (ALP), total bilirubin, neutrophil count, NLR, mean platelet volume (MPV), and FIB4 were independent indices for cirrhosis. Conclusions: In the present study, the role of noninvasive tests in the prediction of liver fibrosis stage and cirrhosis was evaluated in a large cohort of CHB patients. Overall, noninvasive models are gradually becoming more promising. Accordingly, the need for liver biopsy can be reduced with a combination of noninvasive methods in the future.
\end{abstract}

Keywords: Chronic Hepatitis B, Liver, Fibrosis, Noninvasive Models

\section{Background}

Approximately one-third of the world's population is infected with hepatitis B virus (HBV), and 350 - 400 million people are chronic hepatitis B surface antigen (HBsAg) car- riers. The disease has a broad clinical spectrum, ranging from inactive carriers to cirrhosis and hepatocellular carcinoma (HCC). The major aim of treatment is to prevent disease progression to advanced stages, such as cirrhosis and HCC.

Copyright (c) 2017, Hepatitis Monthly. This is an open-access article distributed under the terms of the Creative Commons Attribution-NonCommercial 4.0 International License (http://creativecommons.org/licenses/by-nc/4.0/) which permits copy and redistribute the material just in noncommercial usages, provided the original work is properly cited. 
Diagnosis of hepatic fibrosis is important in the selection of treatment (1). Liver biopsy is the gold standard for the evaluation of hepatic fibrosis stage (2). Although percutaneous liver biopsy is generally considered a safe procedure, it is costly and may increase the risk of complications (3). Biopsy can only represent 1:50.000 of the liver, and its accuracy may be influenced by the techniques and methodological interventions. For instance, fibrosis stage cannot be classified in $20 \%$ of the patients $(4,5)$.

Low compliance of patients due to the invasive nature of biopsy, contraindication of liver biopsy in hemostasis disorders, and limited use of liver biopsy during followup are the limitations of the procedure $(3,6,7)$. The other limitations include the need for static information about the time of liver biopsy for fibrosis, variability of histological findings from the right and left hepatic lobes (considering the biopsy performance), frequent fragmentation of biopsy specimens during biopsy of cirrhotic patients, and finally a fibrosis level lower than expected (8-10).

Many noninvasive methods, including aspartate amino transaminase (AST)/alanine amino transaminase (ALT) ratio (AAR), AST-to-platelet ratio index (APRI), Bonacini cirrhosis discriminant score (CDS), fibrosis-4 (FIB4) index, and age-platelet index (API), have been described to evaluate the degree of hepatic fibrosis (11-15). However, these methods are developed for patients with chronic hepatitis $\mathrm{C}(\mathrm{CHC})$ and produce conflicting results in the prediction of liver fibrosis in patients with chronic hepatitis B (CHB) (16). CHC and CHB are different in terms of virological characteristics, histological changes in the liver, and triggering mechanisms for fibrosis (17). Therefore, it is necessary to evaluate the use of these parameters in patients with CHB.

\section{Objectives}

The aim of this study was to evaluate the relationship between 7 noninvasive models, including AAR, APRI, CDS, API, FIB-4, neutrophil-to-lymphocyte ratio (NLR), and red cell distribution width (RDW)-to-platelet ratio (RPR) in patients with CHB.

\section{Methods}

\subsection{Study Population}

The present research was designed as a retrospective multicenter study, performed at infectious diseases and clinical microbiology and gastroenterology clinics. All patients with CHB undergoing liver biopsy for HBsAg and HBV DNA analysis in more than 6 months, between January 2011 and December 2015, were included in the study. On the other hand, the exclusion criteria were as follows: 1) regular use of alcohol (> $20 \mathrm{~g}$ in a week); 2) use of antiinflammatory drugs; 3 ) decompensated liver disease, renal failure, diabetes mellitus, chronic inflammatory diseases (eg, ulcerative colitis, Crohn's disease, and rheumatoid arthritis), coinfection (eg, HIV, HCV, and HDV), and HCC; 4) other malignancies metabolic and autoimmune liver diseases; and 5) history of liver surgery or transplantation. The medical records of patients undergoing liver biopsy for CHB were evaluated retrospectively. The study was approved by Dumlupinar University clinical research ethics committee.

\subsection{Laboratory Assessment}

The patients' leukocyte count, neutrophil count, lymphocyte, platelet count, hemoglobin, RDW, mean corpuscular volume (MCV), and mean platelet volume (MPV) were recorded, based on the complete blood cell count before biopsy. AST, ALT, albumin, total bilirubin, gamma-glutamyl transferase (GGT), and alkaline phosphatase (ALP) were recorded among biochemical parameters, and the international normalized ratio (INR) was reported among coagulation parameters. HBsAg, anti-HB antibody, HBeAg, antiHBe antibody, HBV DNA, delta-antigen, anti-delta, anti-HIV, and anti-HCV antibodies were also evaluated in viral serological tests.

\subsection{Histological Assessment}

Liver biopsy specimens of the patients were assessed by pathologists in the follow-up location. Liver biopsy specimens, which were assessed according to the histological activity index (HAI) and Ishak fibrosis index, were included in the study (7). The specimens with fibrosis scores of 0 2 were classified as low fibrosis, scores $\geq 3$ were classified as significant fibrosis, and scores of 5 - 6 were defined as cirrhosis. Noninvasive models were compared between patients with low and significant fibrosis, as well as cirrhotic and noncirrhotic patients.

\subsection{Noninvasive Models}

Among the noninvasive models, AAR, API, CDS, FIB4, and APRI were measured for each patient, using different formulas: AAR (De Ritis ratio): AST/ALT; API: age + platelet count (age: $<30$ years, $0 ; 30$ - 39 years, $1 ; 40-49$ years, $2 ; 50$ 59 years, $3 ; 60-69$ years, $4 ; \geq 70$ years, 5); platelet count $\times$ $10^{9} / \mathrm{L}(\geq 225,0 ; 200-224,1 ; 175-199,2 ; 150-174,3 ; 125$ - 149, 4; $<125,5$ ); CDS: platelet count + ALT/AST ratio + INR (platelet count $\times 10^{9} / \mathrm{L}>340,0 ; 280$ - 339, 1; 220 - 279, 2; 160 - 219, 3; $100-159,4 ; 40-99,5 ;<40,6)$ (ALT/AST ratio $>1.7,0 ; 1.2-1.7$, 1; 0.6 - 1.19, 2; < 0.6, 3) (INR < 1.1, 0 ; 1.1 - 1.4, 1; > 1.4, 2); APRI: $\left([\right.$ AST $/$ ULN $] /$ platelet count $\left.\left[\times 10^{9} / \mathrm{L}\right]\right) \times 100$; and FIB4: [age $\times$ 
AST $(\mathrm{U} / \mathrm{L})] /$ platelet count $\left.\left(10^{9} / \mathrm{L}\right) \times \operatorname{ALT}(\mathrm{U} / \mathrm{L}) 1 / 2\right](11-15)$. Moreover, NLR was calculated by dividing the neutrophil count to lymphocyte count in the complete blood cell count, and RPR was calculated by dividing RDW to platelet count (17, 18).

\subsection{Statistical Analysis}

The number cruncher statistical system (NCSS 2007; Kaysville, Utah, USA) was used for the statistical analysis. For the comparison of descriptive results, mean, standard deviation, median, frequency, ratio, minimum, and maximum values were calculated. In addition, regarding quantitative data, student $\mathrm{t}$ test was used for intergroup comparisons of parameters with normal distribution, while Mann-Whitney U test was applied for the intergroup comparisons of parameters without a normal distribution. Diagnosis screening, including sensitivity, specificity, positive predictive value (PPV), negative predictive value (NPV), and receiver operating curve (ROC) analyses, were used to determine the cut-off values for the parameters. Multivariate logistic regression analysis was used to determine the risk factors for fibrosis and cirrhosis. The significance level was set at $\mathrm{P}<0.05$.

\section{Results}

A total of 2520 treatment-naive CHB patients from 40 different centers were included in the study. Overall, $62.6 \%$ of the patients were male, and the mean age was $40.60 \pm$ 12.34 years (minimum, 18 years; maximum, 77 years). The Ishak fibrosis score was $\geq 3$ in $29.8 \%$ of the patients, indicating significant fibrosis (Table 1). According to the comparisons, while no significant difference was found regarding hemoglobin, lymphocyte, RDW, leukocyte, or MPV (P $>0.05$ ), a significant difference was found in the platelet count, albumin, neutrophil count, AST, ALT, INR, GGT, ALP, total bilirubin, and MCV between the groups with low and significant fibrosis $(\mathrm{P}<0.05)$. The prevalence of significant fibrosis was significantly higher in male patients and those with advanced age $(\mathrm{P}<0.05)$.

While albumin level, platelet count, and neutrophil count were significantly lower in the group with significant fibrosis, age, AST, ALT, INR, GGT, ALP, total bilirubin, and MCV were significantly lower in the group with significant fibrosis. API, AST, ALT, INR, albumin, GGT, ALP, and MCV were independent variables for the diagnosis of significant fibrosis in the multiple logistic regression analysis. Two most important independent variables associated with significant fibrosis were INR (odds ratio, 5.234; 95\% CI, 2.73 - 10.04) and API (odds ratio, 1.668; 95\% CI, 1.28 2.16). However, no significant difference was found regarding the mean AAR between the groups $(\mathrm{P}=0.524$; Table 2$)$.
Table 1. The Demographic Characteristics of the Patients

\begin{tabular}{|c|c|}
\hline & Min-Max (Median) \\
\hline Age & $18-77(40)$ \\
\hline Female & $942(27.4)$ \\
\hline Male & $1578(62.6)$ \\
\hline Total bilirubin, mg/dL & $0.1-3.5(0.7)$ \\
\hline AST, U/L & $5-599(35)$ \\
\hline ALT, U/L & $6-1053(52)$ \\
\hline Albumin, g/dL & $2-5.6(4.3)$ \\
\hline GGT, U/L & $1-670(26)$ \\
\hline $\mathbf{A L P}, \mathbf{U} / \mathbf{L}$ & $10-511(78)$ \\
\hline Neutrophil, $\mathbf{1 0}^{3} / \mu \mathrm{L}$ & $0.8-11.6(3.7)$ \\
\hline Lymphocyte, $10^{3} / \mu \mathrm{L}$ & $0.2-7(2.1)$ \\
\hline $\mathbf{H b}, \mathbf{g} / \mathbf{d L}$ & $7.5-18.8(14.6)$ \\
\hline RDW & $6.3-47.7(13.5)$ \\
\hline MCV & $35.8-120.7(87.8)$ \\
\hline MPV & $5.5-22.8(9.2)$ \\
\hline Platelet count, $10^{9} / \mathrm{L}$ & $56-640(215)$ \\
\hline INR & $0.4-3.1(1)$ \\
\hline HBeAg negative & $1966(78)$ \\
\hline HBeAg positive & $554(22)$ \\
\hline HBV DNA $\times 10^{4}, \mathrm{IU} / \mathrm{mL}$ & $0.2-627924(24.9)$ \\
\hline HAI & $0-18(6)$ \\
\hline Fo & $345(13.7)$ \\
\hline F1 & $612(24.3)$ \\
\hline F2 & $810(32.1)$ \\
\hline F3 & $452(17.9)$ \\
\hline F4 & $187(7.4)$ \\
\hline F5 & $96(3,8)$ \\
\hline F6 & $18(0.7)$ \\
\hline
\end{tabular}

Among the noninvasive models, the mean API, APRI, CDS, NLR, FIB4, and RPR were significantly different between the groups with significant and low fibrosis. The ROC curves of these models are presented in Figure 1.

The area under ROC (AUROC) for the noninvasive models of API, APRI, CDS, NLR, FIB4, and RPR were 0.629, 0.688, $0.609,0.470,0.681$, and 0.596 , respectively among CHB patients. The cut-off point for NLR in discrimination of low and significant fibrosis was $\leq 1.63$. Moreover, the cut-off points for API, CDS, APRI, FIB4, and RPR, according to the fibrosis scores, were $>3,5,0.54,1.19$, and 0.07 , respectively. The optimal cut-off point, sensitivity, specificity, PPV, and NPV of 6 noninvasive models, significant in the discrimi- 
Table 2. Group Comparisons and Logistic Regression Analysis of Patients with CHB According to Fibrosis ${ }^{\mathrm{a}}$

\begin{tabular}{|c|c|c|c|c|c|}
\hline & \multicolumn{3}{|c|}{ Group Comparisons } & \multicolumn{2}{|c|}{ Logistic Regression Analysis } \\
\hline & Low Fibrosis (n, 1767) & Significant Fibrosis (n, 753) & PValue & Univariate OR(95\% CI) & Multivariate OR(95\% CI) \\
\hline Age & $39.29 \pm 11.92$ & $43.67 \pm 12.76$ & $0.001^{\mathrm{b}}$ & $1.029(1.022-1.037)$ & \\
\hline Gender (male) & $1072(60.7)$ & $506(67.2)$ & $0.002^{\mathrm{b}}$ & $1,328(1.100-1.589)$ & \\
\hline Platelet count & $228.03 \pm 62.76$ & $206.98 \pm 68.46$ & $0.001^{\mathrm{b}}$ & $0.995(0.993-0.996)$ & \\
\hline AST & $31(23-48)$ & $48(31-89)$ & $0.001^{b}$ & $1.011(1.009-1.013)$ & $1.005(1.001-1.010)$ \\
\hline ALT & $45.5(24-80)$ & $67(40-128)$ & $0.001^{\mathrm{b}}$ & $1.004(1.003-1.005)$ & $0.998(0.995-1.000)$ \\
\hline INR & $1(1-1.1)$ & $1.1(1-1.2)$ & $0.001^{b}$ & $13.825(8.068-23.691)$ & $5.234(2.729-10.040)$ \\
\hline Albumin & $4.29 \pm 0.43$ & $4.16 \pm 0.47$ & $0.001^{\mathrm{b}}$ & $0.534(0.439-0.649)$ & $0.719(0.557-0.927)$ \\
\hline GGT & $24(16-36)$ & $33(21-54)$ & $0.001^{\mathrm{b}}$ & $1.013(1.009-1.016)$ & $1.003(1.001-1.006)$ \\
\hline ALP & $75(60-91)$ & $85(67-109)$ & $0.001^{b}$ & $1.009(1.007-1.012)$ & $1.006(1.003-1.009)$ \\
\hline Total bilirubin & $0.6(0.4-0.9)$ & $0.7(0.5-1)$ & $0.001^{b}$ & $1.782(1.446-2.197)$ & \\
\hline HBeAg positive & $375(21.2)$ & $181(24.1)$ & 0.112 & $1.178(0.962-1.443)$ & \\
\hline HBV DNA $\times 10^{4}$ & $10.2(1.1-344.3)$ & $174.4(8.3-1685)$ & $0.001^{\mathrm{b}}$ & $1.000(1.000-1.000)$ & \\
\hline $\mathbf{H b}$ & $14.42 \pm 1.72$ & $14.41 \pm 1.66$ & 0.855 & $0.995(0.947-1.047)$ & \\
\hline Leukocyte & $6.8(5.6-8)$ & $6.6(5.5-7.8)$ & 0.051 & $0.964(0.919-1.010)$ & \\
\hline Neutrophil & $3.8(3-4.8)$ & $3.5(2.8-4.5)$ & $0.001^{b}$ & $0.892(0.837-0.950)$ & \\
\hline Lymphocyte & $2.1(1.7-2.6)$ & $2.1(1.7-2.6)$ & 0.452 & $0.957(0.845-1.085)$ & \\
\hline RDW & $13.4(12.8-14.4)$ & $13.7(12.8-14.7)$ & 0.061 & $0.989(0.955-1.025)$ & \\
\hline MPV & $9.31 \pm 1.74$ & $9.42 \pm 1.83$ & 0.164 & $1.035(0.986-1.085)$ & \\
\hline MCV & $87.3(84.1-90.4)$ & $88.8(85.3-91.9)$ & $0.001^{b}$ & $1.051(1.035-1.067)$ & $1.017(1.00-1.036)$ \\
\hline API & $2(1-4)$ & $3(2-5)$ & $0.001^{b}$ & $1.272(1.220-1.327)$ & $1.668(1.286-2.164)$ \\
\hline APRI & $0.4(0.2-0.6)$ & $0.6(0.4-1.1)$ & $0.001^{b}$ & $2.403(2.063-2.799)$ & \\
\hline NLR & $1.8(1.4-2.3)$ & $1.7(1.3-2.3)$ & $0.021^{\mathrm{c}}$ & $0.914(0.834-1.002)$ & \\
\hline RPR & $0.06(0.05-0.07)$ & $0.07(0.06-0.09)$ & $0.001^{b}$ & $2,452(1.985-3.027)$ & \\
\hline FIB4 & $0.8(0.6-1.2)$ & $1.3(0.8-2.1)$ & $0.001^{b}$ & $2.040(1.827-2.276)$ & \\
\hline CDS & $4.85 \pm 1.42$ & $5.41 \pm 1.46$ & $0.001^{\mathrm{b}}$ & $1.319(1.240-1.404)$ & \\
\hline AAR & $0.7(0.6-1)$ & $0.7(0.6-0.9)$ & 0.524 & $1.050(0.857-1.287)$ & \\
\hline
\end{tabular}

${ }^{a}$ Values are presented as mean \pm SD, No. (\%) or median (25- 75 quartiles).

${ }^{\mathrm{b}} \mathrm{P}<0.01$.

${ }^{\mathrm{c}} \mathrm{P}<0.05$.

nation of low and significant fibrosis (API, APRI, CDS, NLR, FIB4, and RPR), are presented in Table 3.

The comparison of groups with and without cirrhosis indicated that hemoglobin, leukocyte, and lymphocyte levels were not significantly different between the groups ( $\mathrm{P}>0.05$ ). However, there was a significant difference regarding the platelet count, albumin level, neutrophil count, AST, ALT, INR, GGT, ALP, total bilirubin, HAI, MCV, and RDW between the groups $(\mathrm{P}<0.05)$. All the noninvasive models (API, APRI, AAR, CDS, NLR, RPR, and FIB4) were significant in the discrimination of cirrhosis $(\mathrm{P}<0.05)$. The ROC curves of noninvasive models for the prediction of cir- rhosis are shown in Figure 2. The prevalence of cirrhosis was significantly higher in male patients and those with advanced age $(\mathrm{P}<0.05$; Table 4$)$.

While albumin, platelet count, and neutrophil count were found to be lower in the cirrhotic group, compared to the noncirrhotic group, age, AST, ALT, INR, GGT, ALP, total bilirubin, and MCV were significantly higher in the cirrhotic group ( $\mathrm{P} \leq 0.05)$. In the multiple logistic regression analysis, CDS, albumin, ALP, total bilirubin, neutrophil count, NLR, MPV, and FIB4 were independent risk factors for cirrhosis. The most important independent factor for cirrhosis was FIB4 (odds ratio, 2.349; 95\% CI, 1.31 - 4.199), fol- 
Korkmaz P et al.

Table 3. The Sensitivity, Specificity, Predictive Values, and AUROC of Noninvasive Models (API, CDS, APRI, NLR, FIB4, and RPR) for the Prediction of Significant Fibrosis

\begin{tabular}{|c|c|c|c|c|c|c|c|c|c|}
\hline & \multicolumn{5}{|c|}{ Diagnostic Test } & \multicolumn{3}{|c|}{ ROC Curve } & \multirow[t]{2}{*}{ P Value } \\
\hline & Cut-off & Sensitivity & Specificity & PPV & NPV & Area & SE & $95 \% \mathrm{CI}$ & \\
\hline API & $>3$ & 48.87 & 70.68 & 41.49 & 76.47 & 0.629 & 0.013 & $0.604-0.653$ & $0.001^{\mathrm{a}}$ \\
\hline $\operatorname{CDS}$ & $>5$ & 48.47 & 67.80 & 39.04 & 75.57 & 0.609 & 0.013 & $0.584-0.633$ & $0.001^{\mathrm{a}}$ \\
\hline APRI & $>0.54$ & 56.57 & 71.41 & 45.71 & 79.45 & 0.688 & 0.012 & $0.667 \cdot 0.713$ & $0.001^{\mathrm{a}}$ \\
\hline NLR & $\leq 1.63$ & 46.75 & 59.72 & 33.05 & 72.50 & 0.470 & 0.013 & $0.445-0.496$ & $0.022^{b}$ \\
\hline FIB4 & $>1.19$ & 55.25 & 74.46 & 47.93 & 79.64 & 0.681 & 0.012 & $0.658-0.706$ & $0.001^{\mathrm{a}}$ \\
\hline RPR & $>0.07$ & 40.33 & 75.47 & 40.78 & 75.13 & 0.596 & 0.013 & $0.571-0.622$ & $0.001^{\mathrm{a}}$ \\
\hline
\end{tabular}

$\mathrm{b}_{\mathrm{P}}<0.05$.

Table 4. Group Comparisons and Logistic Regression Analysis of Patients with CHB for the Prediction of Cirrhosis ${ }^{\mathrm{a}}$

\begin{tabular}{|c|c|c|c|c|c|}
\hline & \multicolumn{3}{|c|}{ Group Comparisons } & \multicolumn{2}{|c|}{ Logistic Regression Analysis } \\
\hline & Noncirrhotic (n, 2406) & $\operatorname{Cirrhotic}(\mathbf{n}, 114)$ & PValue & Univariate $\mathrm{OR}(95 \% \mathrm{CI})$ & Multivariate OR(95\% CI) \\
\hline Age & $40.20 \pm 12.16$ & $48.96 \pm 13.17$ & $0.001^{b}$ & $1.057(1.041-1.074)$ & \\
\hline Gender (male) & $1496(62.2)$ & $82(71.9)$ & $0.035^{\mathrm{c}}$ & $1.559(1.027-2.365)$ & \\
\hline Platelet count & $223.95 \pm 64.37$ & $175.25 \pm 65.91$ & $0.001^{b}$ & $0.985(0.981-0.989)$ & \\
\hline AST & $34(24-57)$ & $59.5(37-101)$ & $0.001^{b}$ & $1.007(1.004-1.009)$ & \\
\hline ALT & $51(27-93)$ & $68(41.8-133)$ & $0.001^{\mathrm{b}}$ & $1.002(1.001-1.004)$ & \\
\hline INR & $1(1-1.1)$ & $1.1(1-1.2)$ & $0.001^{\mathrm{b}}$ & $2.020(1.094-3.731)$ & \\
\hline Albumin & $4.26 \pm 0.43$ & $3.92 \pm 0.53$ & $0.001^{\mathrm{b}}$ & $0.248(0.173-0.354)$ & $0.411(0.243-0.693)$ \\
\hline GGT & $25(17-41)$ & $41(26-70)$ & $0.001^{\mathrm{b}}$ & $1.006(1.003-1.009)$ & \\
\hline ALP & $77.5(62-95)$ & $84(67-115.5)$ & $0.007^{\mathrm{b}}$ & $1.008(1.004-1.011)$ & $1.005(1.000-1.010)$ \\
\hline Total bilirubin & $0.6(0.5-0.9)$ & $0.9(0.7-1.2)$ & $0.001^{\mathrm{b}}$ & $3.019(2.156-4.227)$ & $1.612(0.934-2.782)$ \\
\hline HBeAg positive & $525(21.8)$ & $31(27.2)$ & 0.176 & $1.339(0.876-2.045)$ & \\
\hline HBV DNA $\times 10^{4}$ & $21.2(1.6-558.6)$ & $221.7(20-2492.5)$ & $0.001^{b}$ & $1.000(1.000-1.000)$ & \\
\hline Hb & $14.42 \pm 1.70$ & $14.30 \pm 1.82$ & 0.441 & $0.958(0.859-1.068)$ & \\
\hline Leukocyte & $6.7(5.6-8)$ & $6.6(5.5-7.6)$ & 0.347 & $0.946(0.851-1.052)$ & \\
\hline Neutrophil & $3.7(2.9-4.7)$ & $3.4(2.7-4.5)$ & $0.029^{c}$ & $0.879(0.761-1.016)$ & $1.252(1.051-1.491)$ \\
\hline Lymphocyte & $2.1(1.7-2.6)$ & $2.1(1.7-2.7)$ & 0.564 & $1.101(0.843-1.438)$ & \\
\hline RDW & $13.5(12.8-14.5)$ & $13.9(13.1-14.9)$ & $0.009^{\mathrm{b}}$ & $1.027(0.966-1.091)$ & \\
\hline MCV & $87.7(84.3-90.9)$ & $89(85.8-92.4)$ & $0.007^{\mathrm{b}}$ & $1.039(1.006-1.074)$ & \\
\hline MPV & $9.32 \pm 1.76$ & $9.77 \pm 1.89$ & $0.009^{\mathrm{b}}$ & $1.129(1.031-1.238)$ & $1.220(1.055-1.412)$ \\
\hline FIB4 & $0.9(0.6-1.4)$ & $1.9(1.3-3.1)$ & $0.001^{\mathrm{b}}$ & $1.713(1.538-1.908)$ & $2.349(1.314-4.199)$ \\
\hline API & $3(1-4)$ & $5(3-7)$ & $0.001^{\mathrm{b}}$ & $1.579(1.452-1.718)$ & \\
\hline APRI & $0.4(0.3-0.7)$ & $0.9(0.5-1.6)$ & $0.001^{b}$ & $1.692(1.467-1.952)$ & \\
\hline AAR & $0.7(0.6-1)$ & $0.8(0.6-1)$ & $0.020^{\mathrm{C}}$ & $1.475(1.085-2.005)$ & \\
\hline CDS & $4.97 \pm 1.45$ & $5.86 \pm 1.39$ & $0.001^{\mathrm{b}}$ & $1.564(1.361-1.797)$ & $1.259(1.056-1.501)$ \\
\hline NLR & $1.8(1.4-2.3)$ & $1.6(1.3-2.2)$ & $0.046^{c}$ & $0.895(0.719-1.116)$ & $2.018(1.162-3.503)$ \\
\hline RPR & $0.06(0.05-0.08)$ & $0.08(0.07-0.11)$ & $0.001^{\mathrm{b}}$ & $5,031(3.420-7.400)$ & \\
\hline
\end{tabular}

${ }^{\mathrm{a}}$ Values are presented as mean $\pm \mathrm{SD}$, No. (\%), or median (25 - 75 quartiles).

${ }^{\mathrm{b}} \mathrm{P}<0.01$.

${ }^{\mathrm{c}} \mathrm{P}<0.05$. 


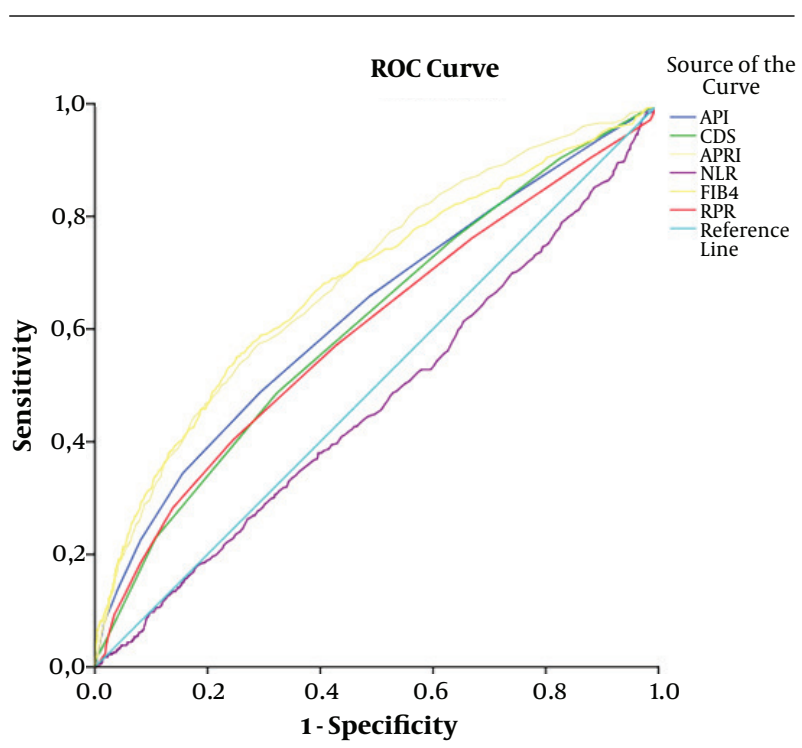

Figure 1. The ROC of Noninvasive Models (API, CDS, APRI, NLR, FIB4, and RPR) in CHB Patients

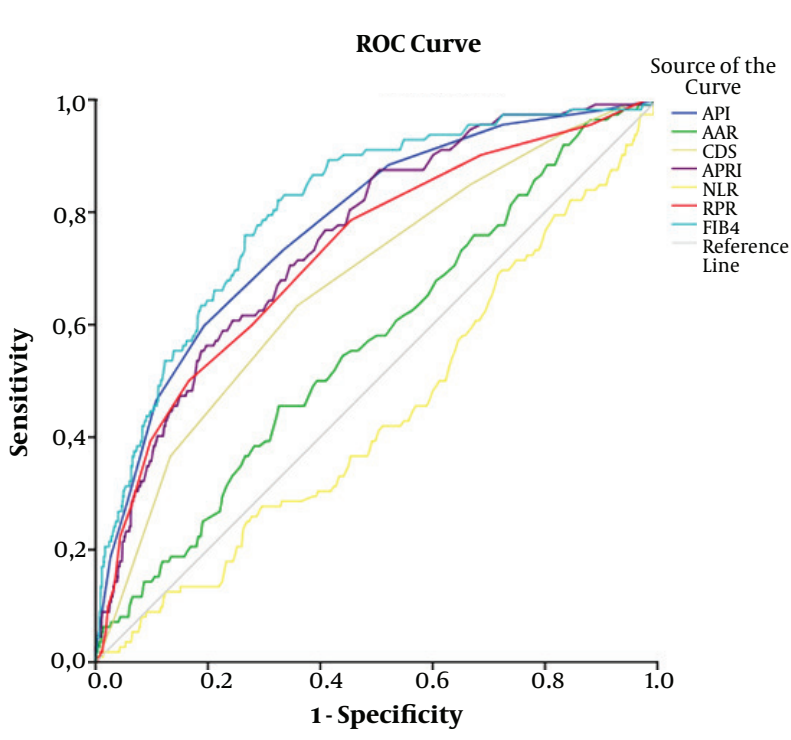

Figure 2. The ROC of Noninvasive Models (API, AAR, CDS, APRI, NLR, FIB4, and RPR) in Cirrhotic Patients lowed by NLR (odds ratio, 2.108; 95\% CI, 1.162 - 3.503; Table 4). The cut-off points for CDS, API, AAR, APRI, FIB4, and RPR in cirrhotic patients were $5,4,0.86,0.4,1.17$, and $>0.08$, respectively. Moreover, the cut-off point for NLR in cirrhotic patients was $\leq 1.62$. The optimum cut-off point, sensitivity, specificity, PPV, and NPV of 7 noninvasive models (API, APRI, CDS, NLR, FIB4, and RPR) in the prediction of cirrhosis are shown in Table 5.

\section{Discussion}

Based on the findings, 6 out of 7 noninvasive models could successfully identify fibrosis in CHB patients without biopsy. The APRI model, which was first investigated by Wai et al. in the staging of fibrosis among CHB patients, showed the most accurate diagnostic value and most powerful predictive value for fibrosis among the models in this study (19). The AUROC of APRI in the prediction of significant fibrosis was 0.688 ; this finding seems to be consistent with the data reported in the literature (20-24). The NPV of APRI in the prediction of fibrosis and cirrhosis was > 90\% in a study by Guzelbulut et al. and 92\% in a study by Celikbilek et al. $(20,22)$. In the present study, the NPVs of APRI for fibrosis and cirrhosis were $79.45 \%$ and $98.84 \%$, respectively. These findings show that APRI is an important indicator, which can exclude fibrosis and cirrhosis and can be used accordingly in $\mathrm{CHB}$ patients.

The cut-off AUROC of FIB4, which is another index used to evaluate liver fibrosis, was 1.19; the sensitivity and specificity of FIB4 for significant fibrosis were $55.25 \%$ and $74.46 \%$, respectively. This cut-off value seems to be consistent with the values reported in the literature $(21,23)$. In a metaanalysis by Xiao et al. the sensitivity and specificity of FIB4 in the prediction of cirrhosis were $87.4 \%$ and $64.7 \%$, respectively (25). In the present study, the sensitivity and specificity of FIB4 in the prediction of cirrhosis were $81.5 \%$ and $67.3 \%$, respectively, based on the AUROC. These findings are consistent with the results obtained by Xiao and colleagues. Moreover, in the present study, the NPVs of FIB4 to exclude significant fibrosis and cirrhosis were $79.64 \%$ and 98.7\%, respectively. Consequently, FIB4 seems to be an important noninvasive assessment tool, which can be used to exclude significant fibrosis, especially cirrhosis.

Lymphocytes play a regulatory role in immune responses and have been shown to influence liver fibrosis in CHB patients (22). NLR is an inexpensive noninvasive model, showing the host's immune responses. Simply, it can be calculated via complete blood cell count parameters. NLR was found to be useful in the evaluation of HCC prognosis and discrimination of nonalcoholic steatohepatitis and significant fibrosis $(26,27)$. In various studies, NLR has been shown to efficiently identify the stage of liver 
Table 5. The Sensitivity, Specificity, PPV, NPV, and AUROC of Noninvasive Models (API, AAR, CDS, APRI, NLR, FIB4, and RPR) for the Prediction of Cirrhosis

\begin{tabular}{|c|c|c|c|c|c|c|c|c|c|}
\hline & \multicolumn{5}{|c|}{ Diagnostic Test } & \multicolumn{3}{|c|}{ ROC Curve } & \multirow[t]{2}{*}{ PValue } \\
\hline & Cut-off & Sensitivity & Specificity & PPV & NPV & Area & SE & $95 \% \mathrm{CI}$ & \\
\hline API & $>4$ & 59.65 & 80.61 & 12.71 & 97.69 & 0.775 & 0.022 & $0.758-0.791$ & $0.001^{\mathrm{a}}$ \\
\hline AAR & $>0.86$ & 44.74 & 67.12 & 6.05 & 96.25 & 0.564 & 0.027 & $0.545-0.584$ & $0.017^{\mathrm{b}}$ \\
\hline $\operatorname{CDS}$ & $>5$ & 64.04 & 64.22 & 7.81 & 97.42 & 0.679 & 0.025 & $0.661-0.698$ & $0.001^{\mathrm{a}}$ \\
\hline APRI & $>0.4$ & 87.72 & 49.56 & 7.60 & 98.84 & 0.752 & 0.022 & $0.735-0.769$ & $0.001^{\mathrm{a}}$ \\
\hline NLR & $\leq 1.62$ & 54.39 & 59.07 & 5.92 & 96.47 & 0.555 & 0.028 & $0.536-0.575$ & $0.047^{\mathrm{b}}$ \\
\hline FIB4 & $>1.17$ & 81.58 & 67.37 & 10.58 & 98.72 & 0.804 & 0.021 & $0.788-0.819$ & $0.001^{\mathrm{a}}$ \\
\hline RPR & $>0.08$ & 50.00 & 83.42 & 12.53 & 97.23 & 0.728 & 0.025 & $0.710-0.745$ & $0.001^{a}$ \\
\hline
\end{tabular}

fibrosis among CHB patients and can be used to demonstrate liver fibrosis $(18,28)$. On the contrary, some studies have indicated no relationship between NLR and cirrhosis and liver fibrosis (22). In the present study, NLR could be used in the discrimination of both significant fibrosis and cirrhosis. Analysis of the relationships, especially between NLR and cirrhosis, was performed using multiple regression analysis, and NLR was determined to be an independent factor for cirrhosis. NLR also showed the greatest value effect, following FIB4 among multiple factors for cirrhosis. However, the efficiency of NLR in the identification of liver fibrosis should be supported in future studies.

RDW is an indicator of variability in erythrocyte volume in circulation. In addition, it is used for the differential diagnosis of anemia (16). The increase in RDW has been shown to be proportional to the severity of liver damage. Thrombocytopenia is one of the well-known markers of severe liver injury. RPR is the ratio of RDW to platelet count and can be calculated according to the complete blood cell count parameters, such as NLR. In the literature, some studies have used RPR in staging of liver fibrosis $(16,29,30)$. Chen et al. reported RPR as a cost-effective and easily measurable method and found it to be superior to AAR, FIB4, and APRI in the prediction of cirrhosis (16). In the present study, AUROC of RPR for significant fibrosis and cirrhosis was 0.596 and 0.728 , respectively. According to the present findings, RPR was a more valuable parameter in the diagnosis of significant fibrosis in comparison with cirrhosis among CHB patients.

CDS is another model in which liver fibrosis is evaluated noninvasively. For the first time, in a study by Bonacini et al. a positive correlation was found between fibrosis score and CDS in CHC patients. In their study, the cut-off value, sensitivity, and specificity of CDS for advanced fibrosis or cirrhosis were $>8,46 \%$, and $98 \%$, respectively (11). Overall, the number of studies indicating a relationship between CDS and fibrosis staging is limited in CHB patients. While CDS was insufficient in the diagnosis of liver fibro- sis in some studies, it could be used to diagnose fibrosis in several other studies $(21,24,30)$. In the present study, the cut-off value and NPV of CDS for low and significant fibrosis in cirrhotic and noncirrhotic patients were $>5,75.57 \%$, and $97.42 \%$, respectively.

According to the present results, CDS is efficient in the discrimination of low fibrosis, significant fibrosis, and cirrhosis. However, since a higher NPV was obtained in the discrimination of cirrhosis, it is more successful in the evaluation of cirrhosis. Since no previous studies in the literature have evaluated the use of CDS in the discrimination of cirrhosis among $\mathrm{CHB}$ patients, the present findings can shed light on future studies.

For the first time, Poynard et al. evaluated API as a model for fibrosis in CHC patients (12). This model has been shown to be a good predictive index for liver fibrosis stage in many studies $(24,30)$. In the present study, AUROC for API was 0.629 , which is consistent with the literature. On the contrary, Erdogan et al. reported that API is ineffective in the discrimination of significant fibrosis (21). Moreover, in an extensive meta-analysis, API was a successful noninvasive model for cirrhosis and NPV was 99.1\% (25). Moreover, in the present study, the NPV of API was 97.69\% for cirrhosis and API was an effective model.

In addition, AAR is a common index for identifying the underlying liver disease. AAR is generally $>2$ in alcoholic liver disease and $<1$ in chronic hepatitis and chronic cholestatic syndrome (24). In the present study, AAR was ineffective in differentiation of low fibrosis from significant fibrosis. There are studies supporting our results in the literature $(21,24,31)$. In some studies, AAR is a parameter, used to identify cirrhosis rather than fibrosis (23). Similarly, we showed that AAR is an index for cirrhosis (Table 4).

INR is the most important indirect and noninvasive index for the prediction of hepatic cirrhosis. ALT, AST, and platelet count are correlated with liver cell death. In liver damage, an increase in PT/INR ratio and a decrease in albumin level have been observed (31). In a study on the 
role of laboratory tests in the identification of fibrosis in CHB patients, platelet count, AST, ALT, GGT, ALP, total bilirubin, albumin, and INR were compared between patients with liver fibrosis stages $0-1$ and 2 - 4; these parameters were found to be significantly different between the groups with respect to fibrosis stage (32). In another study, age, INR, AST, and GGT were associated with liver fibrosis stage (21). In addition, in our study, the association between fibrosis and parameters such as age, albumin level, platelet count, HBV DNA, neutrophil count, AST, ALT, INR, GGT, ALP, total bilirubin, and MCV was investigated. AST, ALT, INR, albumin, GGT, ALP, and MCV were independent predictors of fibrosis in the regression analysis.

In patients with significant liver fibrosis, platelet count decreases due to reduced thrombopoietin production and increased platelet destruction (14). In this regard, conflicting results have been obtained in studies investigating the association between platelet count and fibrosis $(2,8,14$, $15,21,22)$. In addition, in our study, while platelet count was negatively correlated with fibrosis stage in the univariate analysis, it was not an independent parameter in the regression analysis. Similarly, the association between platelet count and cirrhosis was investigated. The platelet count was valuable in identifying cirrhosis in the univariate analysis, whereas it was not significant in the regression analysis. Based on the analyses, further clinical studies are required to clarify the efficiency of platelet count in the identification of fibrosis or cirrhosis alone.

The present study included the largest cohort of patients and investigated all noninvasive models in order to examine liver fibrosis stage and cirrhosis in the literature. The most important limitation of this study is the retrospective design; therefore, assessment by different pathologists was required for fibrosis staging. On the other hand, use of a similar method by all pathologists and highquality standards of Ishak system are the strengths of this study, despite its retrospective design. Additionally, inclusion of patients from different centers is important in representing the general population. Sharing data of one center in relevant studies is reported as limitation of the study and it is stated that data obtained should also be validated by multicenter studies $(19,31)$. The present study is also important in substantiating this validation.

In conclusion, in the present study, the role of noninvasive tests in the prediction of liver fibrosis stage and cirrhosis was evaluated in a large cohort of $\mathrm{CHB}$ patients. Six out of seven noninvasive models for fibrosis stage were efficient in the prediction of significant liver fibrosis. AAR was an insufficient model in staging fibrosis. All 7 noninvasive models were found to be predictive of cirrhosis. FIB4 and APRI were the noninvasive models with the highest diagnostic accuracies for fibrosis stage, while FIB4 and API were the noninvasive models with the highest diagnostic accuracy for cirrhosis. Liver biopsy is currently the gold standard for staging hepatic fibrosis; nevertheless, its status can change in the future. Noninvasive models are gradually becoming more promising, and the need for liver biopsy can be reduced with combinations of noninvasive methods in the future.

\section{Acknowledgments}

Hereby, the authors would like to thank Emire Bor for the statistical analysis of the manuscript.

\section{Footnotes}

Conflicts of Interest: The authors declare no conflicts of interest and no funding.

Funding/Support: No funding support.

\section{References}

1. European Association For The Study Of The L. EASL clinical prac tice guidelines: Management of chronic hepatitis B virus infection. J Hepatol. 2012;57(1):167-85. doi: 10.1016/j.jhep.2012.02.010. [PubMed: 22436845].

2. Zeng MD, Lu LG, Mao YM, Qiu DK, Li JQ, Wan MB, et al. Prediction of significant fibrosis in HBeAg-positive patients with chronic hepatitis B by a noninvasive model. Hepatology. 2005;42(6):1437-45. doi: 10.1002/hep.20960. [PubMed: 16317674].

3. Cadranel JF, Rufat P, Degos F. Practices of liver biopsy in France: results of a prospective nationwide survey. For the Group of Epidemiology of the French Association for the Study of the Liver (AFEF). Hepatology. 2000;32(3):477-81. doi: 10.1053/jhep.2000.16602. [PubMed: 10960438]

4. Siddique I, El-Naga HA, Madda JP, Memon A, Hasan F. Sampling variability on percutaneous liver biopsy in patients with chronic hepatitis C virus infection. Scand J Gastroenterol. 2003;38(4):427-32. [PubMed: 12739716].

5. Regev A, Berho M, Jeffers LJ, Milikowski C, Molina EG, Pyrsopoulos NT, et al. Sampling error and intraobserver variation in liver biopsy in patients with chronic HCV infection. Am J Gastroenterol. 2002;97(10):2614-8. doi: 10.1111/j.1572-0241.2002.06038.x. [PubMed: 12385448].

6. Standish RA, Cholongitas E, Dhillon A, Burroughs AK, Dhillon AP. An appraisal of the histopathological assessment of liver fibrosis. Gut. 2006;55(4):569-78. doi: 10.1136/gut.2005.084475. [PubMed: 16531536].

7. Ishak K, Baptista A, Bianchi L, Callea F, De Groote J, Gudat F, et al. Histological grading and staging of chronic hepatitis. J Hepatol. 1995;22(6):696-9. [PubMed: 7560864].

8. Lok AS, Ghany MG, Goodman ZD, Wright EC, Everson GT, Sterling $\mathrm{RK}$, et al. Predicting cirrhosis in patients with hepatitis $C$ based on standard laboratory tests: results of the HALT-C cohort. Hepatology. 2005;42(2):282-92. doi: 10.1002/hep.20772. [PubMed: 15986415].

9. Pinzani M, Rombouts K, Colagrande S. Fibrosis in chronic liver diseases: diagnosis and management. J Hepatol. 2005;42 Suppl(1):S2236. doi:10.1016/j.jhep.2004.12.008. [PubMed: 15777570].

10. Hsieh YY, Tung SY, Lee $\mathrm{K}$, Wu CS, Wei KL, Shen $\mathrm{CH}$, et al. Routine blood tests to predict liver fibrosis in chronic hepatitis C. World J Gastroenterol. 2012;18(8):746-53. doi: 10.3748/wjg.v18.i8.746. [PubMed: 22371634]. 
11. Bonacini M, Hadi G, Govindarajan S, Lindsay KL. Utility of a discriminant score for diagnosing advanced fibrosis or cirrhosis in patients with chronic hepatitis C virus infection. Am J Gastroenterol. 1997;92(8):1302-4. [PubMed: 9260794].

12. Poynard T, Bedossa P. Age and platelet count: a simple index for predicting the presence of histological lesions in patients with antibodies to hepatitis C virus. METAVIR and CLINIVIR Cooperative Study Groups. J Viral Hepat. 1997;4(3):199-208. [PubMed: 9181529].

13. Giannini E, Botta F, Fasoli A, Ceppa P, Risso D, Lantieri PB, et al. Progressive liver functional impairment is associated with an increase in AST/ALT ratio. Dig Dis Sci.1999;44(6):1249-53. [PubMed: 10389705].

14. Wai CT, Greenson JK, Fontana RJ, Kalbfleisch JD, Marrero JA, Conjeevaram HS, et al. A simple noninvasive index can predict both significant fibrosis and cirrhosis in patients with chronic hepatitis C. Hepatology. 2003;38(2):518-26. doi: 10.1053/jhep.2003.50346. [PubMed: 12883497].

15. Sterling RK, Lissen E, Clumeck N, Sola R, Correa MC, Montaner J, et al. Development of a simple noninvasive index to predict significant fibrosis in patients with HIV/HCV coinfection. Hepatology. 2006;43(6):1317-25. doi: 10.1002/hep.21178. [PubMed:16729309].

16. Chen B, Ye B, Zhang J, Ying L, Chen Y. RDW to platelet ratio: a novel noninvasive index for predicting hepatic fibrosis and cirrhosis in chronic hepatitis B. PLoS One. 2013;8(7). e68780. doi: 10.1371/journal.pone.0068780. [PubMed: 23874760].

17. Lee IC, Chan CC, Huang YH, Huo TI, Chu CJ, Lai CR, et al. Comparative analysis of noninvasive models to predict early liver fibrosis in hepatitis B e Antigen-negative Chronic Hepatitis B. J Clin Gastroenterol.2011;45(3):278-85. doi: 10.1097/MCG.0b013e3181dd5357. [PubMed: 20505530].

18. Kekilli M, Tanoglu A, Sakin YS, Kurt M, Ocal S, Bagci S. Is the neutrophil to lymphocyte ratio associated with liver fibrosis in patients with chronic hepatitis B?. World J Gastroenterol. 2015;21(18):5575-81. doi: 10.3748/wjg.v21.i18.5575. [PubMed: 25987782].

19. Wai CT, Cheng CL, Wee A, Dan YY, Chan E, Chua W, et al. Non-invasive models for predicting histology in patients with chronic hepatitis B. Liver Int. 2006;26(6):666-72. doi: 10.1111/j.1478-3231.2006.01287.x. [PubMed: 16842322].

20. Guzelbulut F, Sezikli M, Akkan-Cetinkaya Z, Yasar B, Ozkara S, KurdasOvunc AO. AST-platelet ratio index in the prediction of significant fibrosis and cirrhosis in patients with chronic hepatitis B. Turk J Gastroenterol. 2012;23(4):353-8. [PubMed: 22965506].

21. Erdogan S, Dogan HO, Sezer S, Uysal S, Ozhamam E, Kayacetin S, et al. The diagnostic value of non-invasive tests for the evaluation of liver fibrosis in chronic hepatitis B patients. Scand J Clin Lab Invest. 2013;73(4):300-8. doi: 10.3109/00365513.2013.773592. [PubMed: 23514016].
22. Celikbilek M, Dogan S, Gursoy S, Zararsiz G, Yurci A, Ozbakir O, et al. Noninvasive assessment of liver damage in chronic hepatitis B. World J Hepatol. 2013;5(8):439-45. doi: 10.4254/wjh.v5.i8.439. [PubMed: 24023983].

23. Teshale E, Lu M, Rupp LB, Holmberg SD, Moorman AC, Spradling P, et al. APRI and FIB-4 are good predictors of the stage of liver fibrosis in chronic hepatitis B: the Chronic Hepatitis Cohort Study (CHeCS). J Viral Hepat. 2014;21(12):917-20. doi: 10.1111/jvh.12279. [PubMed: 25131445].

24. Eminler AT, Ayyildiz T, Irak K, Kiyici M, Gurel S, Dolar E, et al. AST/ALT ratio is not useful in predicting the degree of fibrosis in chronic viral hepatitis patients. Eur J Gastroenterol Hepatol. 2015;27(12):1361-6. doi: 10.1097/MEG.0000000000000468. [PubMed: 26352130].

25. Xiao G, Yang J, Yan L. Comparison of diagnostic accuracy of aspartate aminotransferase to platelet ratio index and fibrosis- 4 index for detecting liver fibrosis in adult patients with chronic hepatitis B virus infection: a systemic review and meta-analysis. Hepatology. 2015;61(1):292-302. doi: 10.1002/hep.27382. [PubMed: 25132233].

26. Halazun KJ, Hardy MA, Rana AA, Woodland D, Luyten EJ, Mahadev $S$, et al. Negative impact of neutrophil-lymphocyte ratio on outcome after liver transplantation for hepatocellular carcinoma. Ann Surg. 2009;250(1):141-51. doi: 10.1097/SLA.0b013e3181a77e59. [PubMed: 19561458].

27. Alkhouri N, Morris-Stiff G, Campbell C, Lopez R, Tamimi TA, Yerian L, et al. Neutrophil to lymphocyte ratio: a new marker for predicting steatohepatitis and fibrosis in patients with nonalcoholic fatty liver disease. Liver Int. 2012;32(2):297-302. doi: 10.1111/j.14783231.2011.02639.x. [PubMed: 22097893].

28. Yesil A, Cosgun S, Erdem E, Kochan K, Gunduz F, Gonen C. The relationship between fibrosis level and blood neutrophil to lymphocyte ratio in chronic HBV patients. Age. 2013;38(13):37-14.

29. Lou Y, Wang M, Mao W. Clinical usefulness of measuring red blood cell distribution width in patients with hepatitis B. PLoS One. 2012;7(5). e37644. doi: 10.1371/journal.pone.0037644. [PubMed: 22649548].

30. Taefi A, Huang CC, Kolli K, Ebrahimi S, Patel M. Red cell distribution width to platelet ratio, a useful indicator of liver fibrosis in chronic hepatitis patients. Hepatol Int. 2015;9(3):454-60. doi: 10.1007/s12072015-9638-9. [PubMed: 26088296].

31. Ma J, Jiang Y, Gong G. Evaluation of seven noninvasive models in staging liver fibrosis in patients with chronic hepatitis $B$ virus infection. Eur J Gastroenterol Hepatol. 2013;25(4):428-34. doi: 10.1097/MEG.0b013e32835cb5dd. [PubMed: 23358121].

32. Zeng X, Xu C, He D, Li M, Zhang H, Wu Q, et al. Performance of several simple, noninvasive models for assessing significant liver fibrosis in patients with chronic hepatitis B. Croat Med J. 2015;56(3):272-9. [PubMed: 26088852]. 